\title{
1F1-1
}

\section{Binocular Colour Fusion Limits about Peripheral Visual Field}

\author{
Xiaolin Qin ${ }^{1}$, Mamoru Takamatsu ${ }^{1}$, Yoshio Nakashima ${ }^{1}$, \\ Zoujirou Katoh $^{2}$, Yasuaki Fukuda ${ }^{3}$ \\ ${ }^{1}$ University of Toyama, ${ }^{2}$ Aichi Mizuho College, ${ }^{3}$ Meijo University
}

\begin{abstract}
In this study, we measured the wavelength difference limit for binocular color vision about peripheral visual field, using a three-dimensional display to present dichoptic stimuli to the left and right eye, respectively. The size of stimulus was $2^{\circ}$ arc of visual angle, the wavelength range of the stimuli was selected from $450 \mathrm{~nm}$ to $650 \mathrm{~nm}$, and the brightness of the experimental stimuli was set $15 \mathrm{~cd} / \mathrm{m}^{2}$. The results suggest that color fusion occurs more difficult with central visual field more than peripheral vision. More importantly, it offers the basis of important fundamental data for some important optical instrument, for example the design of optical microscope etc.
\end{abstract}

Keywords: binocular color fusion, three-dimensional display, peripheral visual field

\section{Introduction}

The binocular fusion is only assured when the input difference of dichoptic stimuli to the left and right eye, is relatively slight whether in shape, in brightness, or in color. In the case of binocular color fusion, a question of how much wavelength difference can be permitted before the fusion ceases is an interesting one.

About measurement of wavelength limit for color fusion, many experiments early had been studied. But the research about peripheral vision is lacking because maybe it's difficult to display the correct stimuli to the correct location of peripheral vision. In this experiment, we aim to measure the binocular color limit about peripheral visual field, and to explore how the visual filed influence the wavelength difference limit $\Delta \lambda$.

\section{Method}

\subsection{Apparatus and Stimuli}

A three-dimensional display device was used to present experimental stimuli dichoptically, i.e. one to each eye of the subject (Fig.1). The similar stimuli comprised a pair of cross fixation (Fig 2b)) and a pair of circular patches (Fig 2(c)). The circle diameter of stimuli was set as approximately $2.5 \mathrm{~cm}$, subtending the visual angle $2^{\circ}$. The cross fixation was white cross subtended a visual of $1^{\circ}$ of arc.

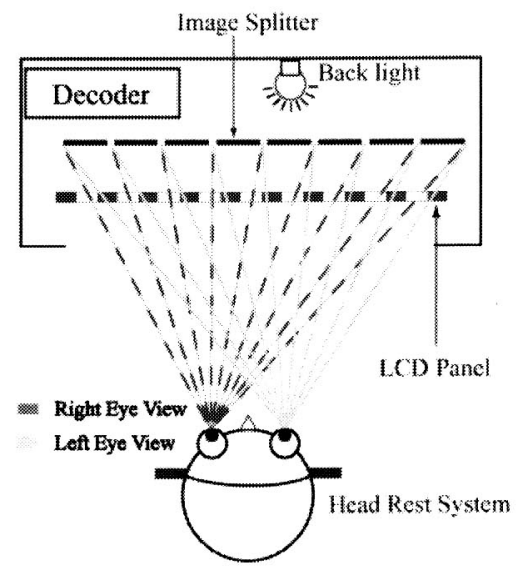

Fig.1 A schematic plan of three-dimension display device (SANYO THD-10P3, display resolution $640 \times 480$, refresh rate $60 \mathrm{HZ}$ ).

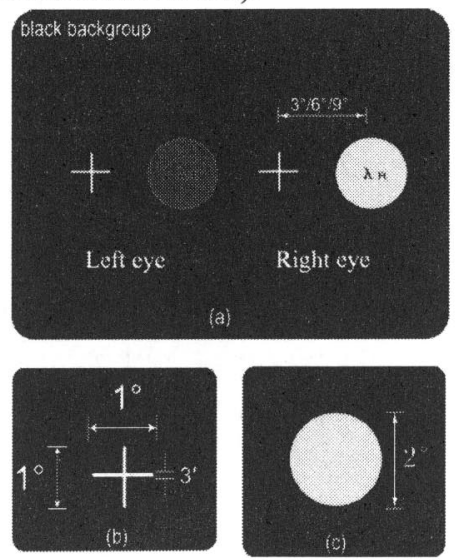

Fig. 2 The subject was presented a left eye view (b) in the left eye and a right eye view (c) in the right eye dichoptically. Brightness was set $15 \mathrm{~cd} / \mathrm{m}^{2}$. 


\subsection{Procedure}

All experiments were carried out in a darkroom. Each subject needed to adapt for the dark environment about $15 \mathrm{~min}$ after the darkroom. The stimuli could be produced or controlled by a computer connected with the three-dimensional display accurately.

Firstly, a white fixation cross, subtending a visual angle of $60 \mathrm{~min}$ arc was presented for 3 seconds, afterward, the fixation dot was kept constant. At the same time the stimulus targets, two circular patches subtending a visual angle of $2^{\circ}$ arc, appeared and lasted for 15 seconds. Then the subject responds fusion or non-fusion. After the response, the experiment did again with another $\lambda R$ until all 41 points are measured.

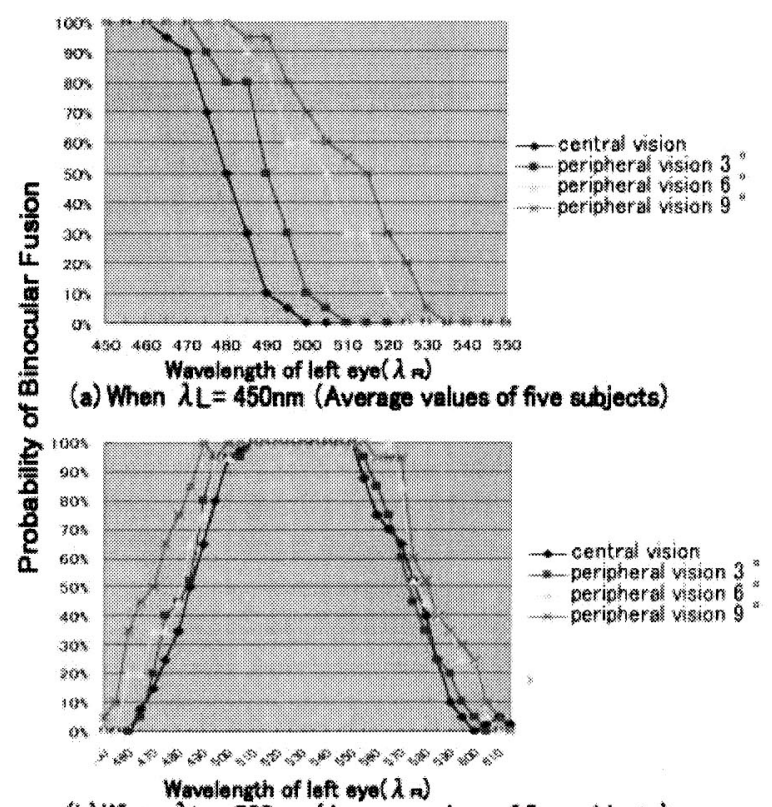

(b) When $\lambda L=520 \mathrm{~nm}$ (Average values of five subjects)

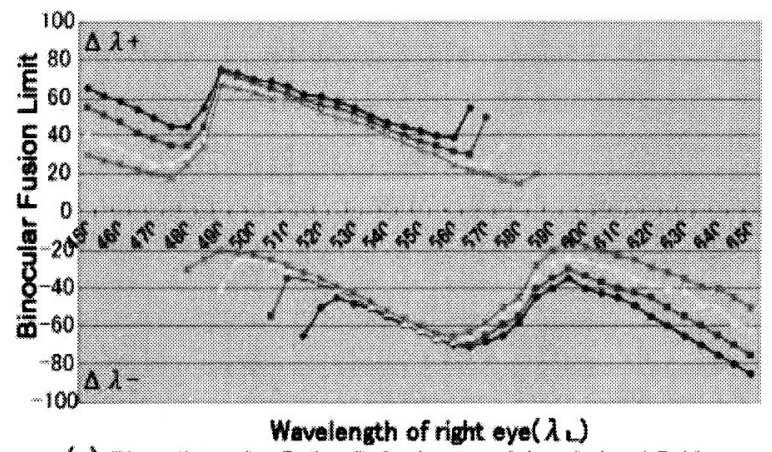

(c) Binocullar color fusion limit about peripheral visual field

Fig.3 Fusion probability curve when $\lambda \mathrm{L}=450 \mathrm{~nm}$ (a) and $520 \mathrm{~nm}$ (b). (c) Binocular fusion limits $\Delta \lambda$ about peripheral visual field as a function of $\lambda \mathrm{L}$ with the fusion probability above $50 \%$.

\section{Results and Discussion}

The results of the experiments reported above lead us to further understand the factors of influencing the color fusion limits $\Delta \lambda$ for each wavelength $\lambda$ by varying the visual field. Two examples are shown in Figure 3(a)(b) in part for $\lambda_{\mathrm{L}}=$ $450 \mathrm{~nm}$ and $520 \mathrm{~nm}$ when the visual filed was adjusted four levels (central vision and peripheral visual field $3^{\circ}, 6^{\circ}, 9^{\circ}$ ), which show clearly that the color fusion forms the inverse relation with the wavelength difference of two eyes.

Figure 3(c) presents some $\Delta \lambda-\lambda$ curves, plus and minus separately, summarizing $\Delta \lambda$ for all subjects under four different conditions with different visual field. We found in Fig 4 there is an increase in the size of the binocular fusional areas with the increase of retinal eccentricity. That is, the disparity limit of central vision is smaller than the limit of peripheral vision with the same stimuli and the same experimental condition.

\section{Conclusion}

The results of the experiments reported above lead us to further understand the factors of influencing the color fusion limits $\Delta \lambda$ for each wavelength $\lambda$ by varying the stimuli luminance and visual angle. More importantly, the quantitative investigations might be useful to offer important fundamental data for the important optical involving dichoptic viewing conditions, for example the design of optical microscope etc.

\section{References}

[1] Howard, I.P. and Rogers, B.J.: Binocular Vision and Steropsis. pp 325-327, (1995)

[2] Johannsen, D.E.: A quantitative study of binocular color vision. Journal of General Psychology, Vol. 4, pp 282-308, (1930)

[3] Ikeda M. and Nakashima Y. (1979) Wavelength difference limit for binocular color fusion. Vision Research. 20. 693-697.

[4] Ikeda M. and Sagawa K. (1979) Binocular color fusion limit. J. opt .Soc. Am 69, 316-321.

[5] Sagawa, K. (1982) Dichoptic color fusion studied with wavelength discrimination. Vision Research, 22,945-952.

[6] Sagawa, K. (1981) Minimum light intensity required for colour rivalry. Vision Research, 22,515-520 